\title{
THEORY OF NONLINEAR FERROMAGNETIC RESONANCE IN THE SAMPLES WITH MAGNETOSTATIC AND EXCHANGE BOUNDARY CONDITIONS IMPOSED
}

\author{
A. Krawiecki and A. Sukiennicki \\ Institute of Physics, Warsaw Technical University \\ Koszykowa 75, 00-662 Warsaw, Poland \\ (Received February 9, 1993)
}

\begin{abstract}
General theory of nonlinear ferromagnetic resonance is presented for samples with the usual magnetostatic and exchange boundary conditions imposed at the sample surface. In such samples the Suhl instabilities and other nonlinear effects occur due to nonlinear interactions of magnetostatic or dipole-exchange modes. All relevant types of interactions are included in the Hamiltonian: with the pumping field, three- and four-mode ones. Analytic calculation of the Suhl thresholds in the three possible types of instabilities in perpendicular and parallel pumping is performed for the sample in the shape of a thin slab.
\end{abstract}

PACS numbers: 76.50.+g, 75.30.Ds 75.70.-i

\section{Introduction}

It is well known that in ferromagnetic resonance experiments, both in perpendicular and parallel pumping, if the applied rf field power exceeds some threshold value, the Suhl threshold [1], time-independent [2] (e.g. appearance of the subsidiary absorption peak, premature saturation of the absorption) and, eventually, time-dependent [3-5] (auto-oscillations, period doubling, intermittency, quasi-periodicity, chaotic transients, chaos etc.) nonlinear behavior of the absorption is observed. These effects appear due to nonlinear interactions among magnetic modes of the sample, excited above their thermal level [1]. If the influence of the sample geometry on the internal magnetic fields and magnetization is neglected, spin-wave modes are appropriate ones. Theoretical investigations have shown $[1,6,7]$ that only interactions involving at most four modes are important, and that in the case of time-dependent signal, though probably an entire manifold of the spin waves is excited, qualitative agreement with the experiment is obtained in a truncated 
model with only some coupled modes and approximately evaluated coupling coefficients (classical "matrix elements" - see below) [8].

Calculation of these coefficients within the spin-wave approximation, valid for bulk ferromagnets, e.g. spheres with the diameter ca. $0.5 \mathrm{~mm}$, commonly utilized in the experiments, is tedious, but straightforward $[1,2,5-7]$. With their values known, it is possible to evaluate the Suhl threshold for a given resonance configuration $[1,2,6,7]$ or to model the time dependence of the absorption in the above-mentioned truncated model (see e.g. $[4,5])$. However, some experiments have been also reported, in which thin films or disks were used as samples. They were performed either as conventional resonance experiments (the sample placed in a cavity), or, especially these reported in the Russian literature, with the rf power provided by a thin metallic antenna, stuck at the sample surface. They included measurements of the Suhl threshold [9-14] and investigation of the time-dependent nonlinear phenomena [15-21]. In a thin slab, the spin-wave approximation loses its validity, because the wavelength of the excited dipole-exchange and magnetostatic modes (true modes of the sample) may be comparable with the sample thickness and the boundary conditions become important, what makes the calculation difficult. On the other hand, in such samples, in a carefully arranged experiment, it is possible to constraint the number of coupled modes to only a few, well separated in energy, ones. If their coupling coefficients are precisely evaluated, one can expect quantitative agreement between the model and the experimental data [17] (e.g. proper values of the Suhl threshold or auto-oscillation frequencies). The main part of this paper is thus devoted to the analytic derivation of the expressions for these coefficients in some special situations, with the influence of the sample shape taken into consideration.

\section{Nonlinear interactions of dipole-exchange and magnetostatic modes in ferromagnetic resonance}

\subsection{General theory}

Existing formulae for the coefficients, describing three- and four-mode interactions in the case, when the boundary conditions are important $[4,17,22-30]$, are not complete and precise enough (see discussion in Sec. 4.), so they are derived in a compact form below. First, a general theory for the sample of arbitrary shape will be considered, and then, the obtained results will be applied to a special case of a thin plate, magnetized parallel or perpendicularly to its surface. The considerations, presented below, are partially based upon the work of Benner and Wiese [4] (who have found three-mode coupling coefficients among the magnetostatic modes in a sphere, using the Landau-Lifshitz equation to describe the motion of the magnetization [1]), but they are performed within the semiclassical Hamiltonian formalism [5-7, 17], and extended to include the four-mode interactions apart from the three-mode ones. The Hamiltonian approach has the advantage of giving the equations of motion in their canonical form, and formulae, obtained for the coefficients of four-mode interactions are more precise than in the method, starting from the Landau-Lifshitz equation [2]. The Hamiltonian of the system has the form 


$$
\mathcal{H}=-M_{0}^{2} \int_{\text {sample }}\left\{M \cdot H_{\text {out }}+\frac{1}{2} M \cdot H_{\mathrm{d}}[M]-\frac{D}{2 M_{0}} \sum_{i=1}^{3}\left(\nabla M_{i}\right)^{2}\right\} \mathrm{d} V .
$$

Here $\boldsymbol{M}$ is the magnetization, $\boldsymbol{H}_{\text {out }}$ is the external field, consisting of the cf part $H_{0}$ and the rf part $h(t), M_{0}$ is the saturation magnetization, $D=2 J S a^{2} / \hbar \gamma$ is the exchange constant, $\gamma$ is the gyromagnetic ratio, and $\boldsymbol{H}_{\mathrm{d}}[\boldsymbol{M}(r, t)]$ denotes the dipolar field of the magnetization in brackets. All the magnetizations and fields are normalized to $M_{0}$. The first term in the Hamiltonian is the interaction energy with the external. field, the second one is the dipolar self-energy and the third one - the exchange energy. Anisotropy effects are not included. In the following, the coordinate system will always have the $z$-axis parallel to the direction of the external cf field $\boldsymbol{H}_{0}$.

A classical version of the Holstein-Primakoff transformation is now used to write the Hamiltonian in terms of the canonical variables $a, a^{*}$

$$
M^{+}=\left[\frac{2 \gamma \hbar}{M_{0}}\right]^{1 / 2}\left[1-\frac{\gamma \hbar}{2 M_{0}} a^{*} a\right]^{1 / 2} a, \quad M_{z}=1-\frac{\gamma \hbar}{M_{0}} a^{*} a .
$$

Here $M^{+}=M_{x}+\mathrm{i} M_{y}, M^{-}=M^{+*}$ and $M_{z}$ form a complex representation of the real magnetization $\boldsymbol{M}$, and the star denotes the complex conjugate.

At that point, the variables $a, a^{*}$ are usually expanded in the Fourier series. In the following, however, the expansion in terms of dipole-exchange or magnetostatic modes will be performed $[4,17,30]$, because they are already fit for the sample shape. These modes form a complete set of solutions to the system of partial differential equations, consisting of the Maxwell equations in the magnetostatic approximation (electromagnetic propagation effects neglected) and the Landau-Lifshitz equation in the linear approximation. If the exchange field is neglected, the solutions are called magnetostatic modes [31]; otherwise, they are called dipole-exchange modes [32]. Henceforth it will be assumed that the magnetization $\boldsymbol{m}$ of any dipole-exchange mode at the sample surface fulfills the pinning boundary conditions [33]

$$
a m+b \frac{\partial m}{\partial n}=0 .
$$

Here $0 \leq a, b \leq 1$ are real constants and $\partial / \partial n$ denotes the normal derivative. After introducing the magnetostatic potential $\psi$ [31], such that $h_{\mathrm{d}}=\nabla \psi$, where $h_{\mathrm{d}}$ is the rf part of the dipolar field, such exchange boundary conditions, together with the common magnetostatic boundary conditions, lead to the characteristic equation, which determines the frequencies of the allowed, in general, complex, solutions $\psi_{k}$ (mode potentials). Henceforth $m_{k}$ and $\boldsymbol{H}_{\mathrm{d}}\left[\boldsymbol{m}_{k}\right]=\nabla \psi_{k}$ denote the mode magnetization and dipolar field, respectively, and $k$ is the mode index. It may be proved that two modes with distinct frequencies, and obeying (3), fulfill the following orthogonality relations $[31,34]$

$$
\begin{aligned}
& \int\left(m_{k}^{-} m_{l}^{+}-m_{l}^{-} m_{k}^{+}\right) \mathrm{d} V=0, \\
& \int\left(m_{k}^{-} m_{l}^{-*}-m_{k}^{+} m_{l}^{+*}\right) \mathrm{d} V=V \delta_{k l} .
\end{aligned}
$$


Here $V$ is the sample volume, $\int \mathrm{d} V$ denotes the integration over $V, \delta_{k l}$ is the Kronecker delta, and $m_{k}^{+}=m_{k x}+\mathrm{i} m_{k y}, m_{k}^{-}=m_{k x}-\mathrm{i} m_{k y}$, as usually. Let us note that Eq. (5) determines the normalization for the modes.

The expansion of $a, a^{*}$ in terms of the dipole-exchange modes is

$$
a(r, t)=V^{-1 / 2} \sum_{k}\left[a_{k}^{*}(t) m_{k}^{+}(r)+a_{k}(t) m_{k}^{-*}(r)\right] .
$$

After inserting Eqs. (6), (2) into (1) (we emphasize that contributions to the dipolar field from both the linear and nonlinear parts of the expression for the magnetization (2) should be retained) and taking into account that for any two magnetizations $m_{1}, m_{2}[4]$

$$
\int m_{1}(r) \cdot H_{\mathrm{d}}\left[m_{2}(r)\right] \mathrm{d} V=\int m_{2}(r) \cdot H_{\mathrm{d}}\left[\boldsymbol{m}_{1}(r)\right] \mathrm{d} V
$$

the Hamiltonian takes on the form of a power series with respect to $a_{k}, a_{k}^{*}$ plus the term, describing the interaction of the magnetization with the pumping field

$$
\mathcal{H}=\text { const }+\mathcal{H}_{\mathrm{p}}+\mathcal{H}_{2}+\mathcal{H}_{3}+\mathcal{H}_{4}
$$

$\mathcal{H}_{\mathrm{p}}$ consists of terms involving the external rf field, $\mathcal{H}_{2}$ consists of quadratic terms in $a_{k}, a_{k}^{*} ; \mathcal{H}_{3}$ of cubic terms and $\mathcal{H}_{4}$ - of quartic terms. Higher-order terms need not be included.

In the following, we shall constraint the discussion to the case of the uniform in space rf pumping field. In the case of perpendicular pumping, one can assume the time dependence of the rf field in the form of $h(t)=h_{T} e_{y} \cos \omega t$, and in the case of parallel pumping $h(t)=h_{z} e_{z} \cos \omega t$, where the amplitudes $h_{T}, h_{z}$ are real. $\mathcal{H}_{\mathrm{p}}$ the pumping term, is then [17]

$$
\begin{aligned}
& \mathcal{H}_{\mathrm{p}}=\hbar^{1 / 2} h_{T} \cos \omega t \sum_{k} I_{k} a_{k}^{*}+\text { c.c. }, \\
& I_{k}=-M_{0}^{3 / 2}\left[\frac{2 \gamma}{V}\right]^{1 / 2} \int m_{k y}(r) \mathrm{d} V,
\end{aligned}
$$

in the case of perpendicular pumping and $[4,34]$

$$
\begin{aligned}
& \mathcal{H}_{\mathrm{p}}=\hbar h_{z} \cos \omega t \sum_{k, l} J_{k l} a_{k}^{*} a_{l}^{*}+\text { c.c. }, \\
& J_{k l}=\frac{\gamma}{V} M_{0} \int m_{k}(r) \cdot m_{l}(r) \mathrm{d} V
\end{aligned}
$$

in the case of parallel pumping. $I_{k}$ and $J_{k l}$ are the coupling coefficients for the interaction between the pumping field and the directly excited modes. In parallel pumping the rf field directly excites pairs of modes with the frequency $\omega / 2$. If the pumping field is strong enough, their amplitudes can grow exponentially in time - this is the parallel pumping instability [2]. In the case of perpendicular pumping usually only one mode, e.g. the uniform one, is strongly excited. When the rf transverse field power exceeds some threshold value (Suhl threshold), the directly pumped mode can decay into two another modes with the frequencies $\omega_{1}+\omega_{2}=\omega$ (the 1st order Suhl instability) [1]. If this process is forbidden by the energy conservation, i.e. the energy gap below the mode spectrum is larger 
than $\omega / 2$, two quanta of this mode can decay into two modes with the frequencies $\omega_{1}+\omega_{2}=2 \omega$ (the 2nd order Suhl instability) [1]. The former process is described by the three-mode interactions, contained in $\mathcal{H}_{3}$, and the later - by the four-mode interactions in $\mathcal{H}_{4}$. This part of the Hamiltonian takes also into account another four-mode interactions, e.g. the coupling among the modes, created in the Suhl instabilities [6]. In the following, like in the S-theory in the spin-wave approximation [7], only the terms in $\mathcal{H}_{\mathbf{4}}$ with two creation and two annihilation "operators" $\left(a_{k}^{*}, a_{k}\right.$, respectively) will be retained.

The remaining part of the Hamiltonian is

$$
\begin{aligned}
& \mathcal{H}_{2}=\sum_{k} \hbar \omega_{k} a_{k}^{*} a_{k}, \\
& \begin{array}{c}
\mathcal{H}_{3}=\sum_{k 1, k 2, k 3} \hbar^{3 / 2} V_{k 1 k 2 k 3} a_{k 1}^{*} a_{k 2} a_{k 3}+\text { c.c. } \\
\quad+\hbar^{3 / 2} U_{k 1 k 2 k 3} a_{k 1} a_{k 2} a_{k 3}+\text { c.c., }
\end{array} \\
& \begin{array}{r}
\mathcal{H}_{4}=\sum_{k 1, k 2, k 3, k 4} \hbar^{2} T_{k 1 k 2 k 3 k 4} a_{k 1}^{*} a_{k 2}^{*} a_{k 3} a_{k 4}, \\
V_{k 1 k 2 k 3}=\frac{1}{2}\left[\frac{2 \gamma}{V}\right]^{3 / 2} M_{0}^{1 / 2} \int \mathrm{d} V\left\{H_{\mathrm{d} z}\left[m_{k 1}\right] m_{k 2}^{*} \cdot m_{k 3}^{*}\right. \\
\left.\quad+H_{\mathrm{d} z}\left[m_{k 2}^{*}\right] m_{k 1} \cdot m_{k 3}^{*}+H_{\mathrm{d} z}\left[m_{k 3}^{*}\right] m_{k 1} \cdot m_{k 2}^{*}\right\} \\
U_{k 1 k 2 k 3}=\frac{1}{6}\left[\frac{2 \gamma}{V}\right]^{3 / 2} M_{0}^{1 / 2} \int \mathrm{d} V\left\{H_{\mathrm{d} z}\left[m_{k 1}^{*}\right] m_{k 2}^{*} \cdot m_{k 3}^{*}\right. \\
\left.\quad+H_{\mathrm{d} z}\left[m_{k 2}^{*}\right] m_{k 1}^{*} \cdot m_{k 3}^{*}+H_{\mathrm{d} z}\left[m_{k 3}^{*}\right] m_{k 1}^{*} \cdot m_{k 2}^{*}\right\}
\end{array}
\end{aligned}
$$

$$
\begin{aligned}
& T_{k 1 k 2 k 3 k 4}=T_{k 1 k 2 k 3 k 4}^{\mathrm{dip}}+T_{k 1 k 2 k 3 k 4}^{\mathrm{ex}}, \\
& T_{k 1 k 2 k 3 k 4}^{\mathrm{dip}}=T_{k 1 k 2 k 3 k 4}^{(1) \operatorname{dip}_{k}}-T_{k 1 k 2 k 3 k 4}^{(2) \operatorname{dip}_{1}}, \\
& T_{k 1 k 2 k 3 k 4}^{(1) \operatorname{dip}}=\frac{1}{2}\left[\frac{\gamma}{2 V}\right]^{2} \sum_{i, j, k, l} \int \mathrm{d} V\left(M_{i} \cdot M_{j}\right)\left(H_{d}\left[M_{k}\right] \cdot M_{l}\right) .
\end{aligned}
$$

In the above expression the sequence of subscripts $i, j, k, l$ is a permutation of the set $k 1, k 2, k 3, k 4$ of the mode indices and $M_{i}=m_{i}$ if $i=k 1, k 2$ or $M_{i}=m_{i}^{*}$ if $i=k 3, k 4$.

$$
\begin{aligned}
& T_{k 1 k 2 k 3 k 4}^{(2) \operatorname{dip}}=\left[\frac{\gamma}{V}\right]^{2} \int \mathrm{d} V\left\{\boldsymbol{m}_{k 1} \cdot \boldsymbol{m}_{k 2} H_{\mathrm{d} z}\left[\left(m_{k 3}^{*} \cdot \boldsymbol{m}_{k 4}^{*}\right) \boldsymbol{e}_{z}\right]\right. \\
& \left.+\boldsymbol{m}_{k 1} \cdot \boldsymbol{m}_{k 3}^{*} H_{\mathrm{d} z}\left[\left(m_{k 2} \cdot m_{k 4}^{*}\right) \boldsymbol{e}_{z}\right]+m_{k 2} \cdot m_{k 3}^{*} H_{\mathrm{d} z}\left[\left(m_{k 1} \cdot \boldsymbol{m}_{k 4}^{*}\right) \boldsymbol{e}_{z}\right]\right\} \\
& T_{k 1 k 2 k 3 k 4}^{\text {ex }}=T_{k 1 k 2 k 3 k 4}^{(1) \operatorname{ex}}+T_{k 1 k 2 k 3 k 4}^{(2) \text { ex }} .
\end{aligned}
$$

An expression for $T_{k 1 k 2 k 3 k 4}^{(1) \text { ex }}$ may be obtained from (18) simply by writing $\nabla^{2} M_{k}$ instead of $\boldsymbol{H}_{\mathrm{d}}\left[M_{k}\right]$, and multiplying by the constant $\left(D / M_{0}\right)$, and 


$$
\begin{aligned}
& T_{k 1 k 2 k 3 k 4}^{(2) e x}=\frac{D}{M_{0}}\left[\frac{\gamma}{V}\right]^{2} \int \mathrm{d} V\left\{\nabla\left(\boldsymbol{m}_{k 1} \cdot \boldsymbol{m}_{k 2}\right) \cdot \nabla\left(\boldsymbol{m}_{k 3}^{*} \cdot \boldsymbol{m}_{k 4}^{*}\right)\right. \\
& \left.\quad+\nabla\left(\boldsymbol{m}_{k 1} \cdot \boldsymbol{m}_{k 3}^{*}\right) \cdot \nabla\left(\boldsymbol{m}_{k 2} \cdot \boldsymbol{m}_{k 4}^{*}\right)+\nabla\left(\boldsymbol{m}_{k 2} \cdot \boldsymbol{m}_{k 3}^{*}\right) \cdot \nabla\left(\boldsymbol{m}_{k 1} \cdot \boldsymbol{m}_{k 4}^{*}\right)\right\}
\end{aligned}
$$

$U, V, T$ are the three- and four-mode coupling coefficients we have been looking for (Eq. (16) was also derived in [4]). The long expressions, presented above, may be slightly simplified, because e.g. four coefficients $T_{k 1 k 2 k 3 k 4}, T_{k 2 k 1 k 3 k 4}, T_{k 1 k 2 k 4 k 3}$, $T_{k 2 k 1 k 4 k 3}$ appear with identical combinations of $a_{k 1}^{*}, a_{k 2}^{*}, a_{k 3}, a_{k 4}$, only variously ordered. One can then join similar terms together and divide them among four new coefficients to make the notation shorter, but the number of distinct integrals over the sample volume, which must be performed, will not be diminished. In the present notation, the four above-mentioned coupling coefficients are equal to each other and $T_{k 1 k 2 k 3 k 4}=T_{k 4 k 3 k 2 k 1}^{*}$, as expected. Let us note also that the obtained formulae for all coefficients remain valid in the spin-wave approximation. In order to obtain their explicit form in that case, the expansion (6) must be replaced with the Fourier expansion of $a, a^{*}$, and - before inserting it into the Hamiltonian the Bogoliubov transformation must be performed.

It is easily seen from Eqs. $(16-18,20)$ that these expressions may be calculated, if the mode magnetizations are known.-In fact, the task is only to evaluate three-dimensional integrals over the sample volume, what at worst may be performed numerically. A more difficult problem is to evaluate the dipolar fields of the scalar products of the mode magnetizations in Eq. (19). The result usually cannot be obtained analytically and it is the main constraint for the applications of the given formulae to real samples, e.g. spheres. In the case of thin slabs, however, the exact functional form of this dipolar field may be given, owing to the simplicity of the magnetostatic potential for both dipole-exchange and magnetostatic modes in a thin plate. Then, the Suhl thresholds for any of the three above-mentioned instabilities may be calculated as from the spin-wave approximation $[1,2,6-7]$, but, hopefully, with more accuracy.

\subsection{Application to the magnetostatic modes in a thin slab}

In this paragraph, the general results, obtained in the preceding section, will be applied to the special case of a thin ferromagnetic slab, infinite in two dimensions; magnetized parallel or perpendicularly to its surface. Only magnetostatic modes will be considered, because of the relative simplicity of the calculations in comparison with the case of the dipole-exchange modes. One can always arrange the experiment in such a way that only the modes from the lowest surfaces of the energy spectrum are involved in the Suhl instabilities, for example in very thin films, where the exchange interaction shifts the higher-order surfaces considerably upwards [32]. The exchange affects not only the frequencies of the modes, but the magnetostatic potential, too [32]. This can influence the exact numerical results, even if the coupled modes have relatively small wave numbers, and their frequencies are essentially unmodified by the exchange forces. But if one is able to obtain the potential and frequencies of the dipole-exchange modes, calculation similar to that below may be performed, because such a potential differs from the 
magnetostatic mode potential rather not in its functional form, but in the number of constants, determined from the boundary conditions [32].

In the case of a thin slab, magnetized parallel to its surface, the coordinate system has the $x$-axis perpendicular to the sample surface, the $z$-axis parallel to the surface, along the direction of the external cf field. The magnetostatic modes may be indexed with the numbers $k_{x}, q_{y}, q_{z}$, which are the wave numbers in the $x, y, z$ direction. The potential of the mode inside the slab is [35]

$$
\psi(r)=\left[A \sin \left(k_{x} x\right)+B \cos \left(k_{x} x\right)\right] \exp \left[\mathrm{i}\left(q_{y} y+q_{z} z\right)\right] .
$$

If $k_{x}$ is real, this is the volume mode potential, and if $k_{x}$ is purely imaginary the surface mode potential. $A, B$ are constants, determined from the boundary conditions and the normalization (5). From Eq. (21) a vector function $m(x)$ may be obtained, such that the mode magnetization is $\boldsymbol{m}(\boldsymbol{r})=\boldsymbol{m}(\boldsymbol{x}) \exp \left[\mathrm{i}\left(q_{y} y+q_{z} z\right)\right]$.

The dipolar field of the scalar product of the magnetizations of two modes, say, 1 and 2, appearing in Eq. (19), may be calculated with the help of the magnetostatic Green function for the slab [25]. If we denote $q_{1,2}=q_{y 1,2} e_{y}+q_{z 1,2} e_{z}$ and $f_{12}(x)$ is such a function that $m_{1}(r) \cdot m_{2}^{*}(r)=f_{12}(x) \exp \left[\mathrm{i}\left(q_{1}-q_{2}\right) \rho\right](\rho$ is the part of $r$ in the plane of the slab), then the respective dipolar field in Eq. (19) is zero if $q_{1}=q_{2}$ and elsewhere

$$
\begin{aligned}
& H_{\mathrm{d} z}\left\{\boldsymbol{e}_{z} f_{12}(x) \exp \left[\mathrm{i}\left(q_{1}-q_{2}\right) \rho\right]\right\} \\
& \quad=-2 \pi \exp \left[\mathrm{i}\left(\boldsymbol{q}_{1}-\boldsymbol{q}_{2}\right) \rho\right] \int_{-S / 2}^{S / 2} \mathrm{~d} x^{\prime} f_{12}\left(x^{\prime}\right) G\left(x, x^{\prime}, q_{1}, q_{2}\right), \\
& G\left(x, x^{\prime}, q_{1}, q_{2}\right)=\frac{\left(q_{1 z}-q_{2 z}\right)^{2}}{\left|q_{1}-q_{2}\right|} \exp \left(-\left|q_{1}-q_{2}\right|\left|x-x^{\prime}\right|\right) .
\end{aligned}
$$

Here $S$ is the thickness of the slab. The integral in (22) may be evaluated analytically and here it is denoted as $F_{12}(x)$. Then the input from e.g. the second term in brackets in Eq. (19) to $T_{k 1 k 2 k 3 k 4}^{(2) \text { dip }_{12}}$ is

$$
T_{k 1 k 2 k 3 k 4}^{(2) \mathrm{dip}}=-(2 \pi)^{3}\left(\frac{\gamma}{4 \pi^{2} S}\right)^{2} \delta\left(q_{1}+q_{2}-q_{3}-q_{4}\right) \int_{-S / 2}^{S / 2} F_{24}(x) f_{13}(x) \mathrm{d} x .
$$

Here $\delta$ denotes the Dirac delta function; $1, \ldots 4$ are abbreviations for $k 1, \ldots k 4$, and, because the slab is infinite in two dimensions, the volume of the sample $V$ was replaced, also in the normalization (5), by $(2 \pi)^{2} S$. The integral in (24) may be also evaluated analytically. The remaining inputs to $T_{k 1 k 2 k 3 k 4}^{(2) \text { dip }}$ may be calculated in a similar way, and the remaining contributions to the four-mode coupling coefficient - easily evaluated from Eqs. (21) and (18). In our case (exchange effects neglected) the exchange part of this coefficient is, of course, unimportant.

In the case of a perpendicularly magnetized slab, we proceed in the same way. The coordinate system has its $z$-axis perpendicular to the sample surface, and $x, y$ axes - in the plane of the surface. The magnetostatic potential is [36]

$$
\psi(r)=\left[A \exp \left(\mathrm{i} k_{z} z\right)+B \exp \left(-\mathrm{i} k_{z}\right)\right] \exp \left[\mathrm{i}\left(q_{x} x+q_{y} y\right)\right] .
$$


The magnetization of this mode is $m(r)=m(z) \exp \left[\mathrm{i}\left(q_{x} x+q_{y} y\right)\right]$ and we define $q_{1,2}=q_{x 1,2} e_{x}+q_{y 1,2} e_{y}$. If $m_{1}(r) \cdot m_{2}^{*}(r)=f_{12}(z) \exp \left[\mathrm{i}\left(q_{1}-q_{2}\right) \rho\right]$, then, in analogy with Eq. (22)

$$
\begin{aligned}
& H_{\mathrm{d} z}\left\{\boldsymbol{e}_{z} f_{12}(z) \exp \left[\mathrm{i}\left(\boldsymbol{q}_{1}-q_{2}\right) \rho\right]\right\} \\
& =-2 \pi \exp \left[\mathrm{i}\left(\boldsymbol{q}_{1}-q_{2}\right) \rho\right] \int_{-\mathrm{S} / 2}^{\mathrm{S} / 2} \mathrm{~d} z^{\prime} f_{12}\left(z^{\prime}\right) G\left(z, z^{\prime}, q_{1}, q_{2}\right) \\
& G\left(z, z^{\prime}, q_{1}, q_{2}\right)=2 \delta\left(z-z^{\prime}\right)-\left|q_{1}-q_{2}\right| \exp \left(-\left|q_{1}-q_{2}\right|\left|z-z^{\prime}\right|\right)
\end{aligned}
$$

The Dirac delta function in (27) leads to the approximation of the dipolar field of arbitrary magnetization $m(\boldsymbol{r})$ in a perpendicularly magnetized slab $H_{\mathrm{d} z}[m(r)]=$ $-4 \pi m_{z}(z)$. The formula for $T_{k 1 k 2 k 3 k 4}^{(2) \text { dip }}$ may be now obtained in a similar manner as Eq. (24), and all integrations are again analytically performable.

In a thin disk, magnetized perpendicularly to its surface (cylindrical system of coordinates with the $z$-axis perpendicular to the surface) the magnetostatic mode potential is [33]

$$
\psi(r)=A J_{n}\left(k_{\rho} \rho\right) \cos \left(k_{z} z-\frac{1}{2} \eta_{z} z\right) \mathrm{e}^{-\mathrm{in} \phi} .
$$

$J_{n}$ is the Bessel function of integer order $n, \eta_{z}=0$ or 1 for modes even or odd in $z$. The modes are indexed by $n, k_{\rho}, k_{z}$. Expanding $1 /\left|r-r^{\prime}\right|$ in the cylindrical system of coordinates, it is possible to obtain in the first approximation $H_{\mathrm{d} z}\left[\boldsymbol{m}_{z}(r) e_{z}\right]=$ $-4 \pi m_{z}(r)$ [17], in analogy with Eq. (27), but exact calculation of the dipolar fields in Eq. (19) is rather impossible. Thus only "selection rules" for the angular part of the mode potentials are given. The three-mode coupling coefficients (16) are non-zero only if

$$
n_{2}+n_{3}=n_{1}
$$

and the four-mode coupling coefficients $(18,19)$ are non-zero if

$$
n_{1}+n_{2}=n_{3}+n_{4} \text {. }
$$

Equations $(29,30)$ are equivalent to the angular momentum conservation, as the Dirac delta function in Eq. (24) means the momentum conservation. Analogous "selection rules" are valid for the magnetostatic potential of the sphere (cf. $[4,30])$.

\section{Suhl instability thresholds}

The expressions, derived in the previous paragraph, enable us to do the direct calculation of the nonlinear coupling coefficients among the magnetostatic modes in a thin slab. As already mentioned, the Suhl thresholds for any of the three types of instabilities may be then evaluated. This procedure has been widely described by many authors $[1,2,6,7]$, so only the results will be given here. It is assumed that in the parallel pumping instability only one pair of modes (we denote them as $k,-k$, in analogy with the spin-wave theory, to indicate that some selection rules, must be fulfilled) with frequencies $\omega_{k} \approx \omega / 2$, detuning $\Delta \omega_{k}=\omega / 2-\omega_{k} \approx 0$, and phenomenological damping $\eta_{k}$ is directly excited by the rf pumping field above the Suhl threshold. Then the critical value of the rf field amplitude for the occurrence of the instability is

$$
h_{z \mathrm{crit}}=\frac{2\left|\delta_{k}\right|}{\left|J_{k}\right|}
$$


where $J_{k}=J_{k,-k}+J_{-k, k}$ in Eq. (12), $\delta_{k}=\eta_{k}+\mathrm{i} \Delta \omega_{k}$. In the case of both types of the perpendicular pumping instabilities it is assumed that only one mode (we denote it as the zero one) is directly coupled to the rf field with the coupling coefficient $I_{0}$ from (10), detuning $\Delta \omega_{0}$ and damping $\eta_{0}$. In a finite plate it need not be the uniform mode; in fact, many absorption peaks at different frequencies can be seen in the resonance spectrum. This mode then decays into one pair of modes $k,-k$ with the detuning $\Delta \omega_{k}=\omega / 2-\omega_{k}$ or $\Delta \omega_{k}=\omega-\omega_{k}$ for the 1st or 2 nd order Suhl instability, respectively. Then the critical values of the rf field amplitude are: for the 1st order instability

$$
h_{T \text { crit }}^{\mathrm{I}}=\frac{2\left|\delta_{0}\right|\left|\delta_{k}\right|}{\left|I_{0}\right||V|},
$$

where $V=V_{0, k,-k}+V_{0,-k, k}$ from Eq. (16) and for the 2nd order instability

$$
h_{T \text { crit }}^{\mathrm{II}}=\frac{2\left|\delta_{0}\right|}{\left|I_{0}\right|}\left(\frac{\left|\delta_{k}\right|}{|T|}\right)^{1 / 2}
$$

where $T=2\left(T_{k,-k, 0,0}+T_{-k, k, 0,0}\right)$ from Eqs. (18-20). In the latter case, however, it is necessary to consider the influence of the virtual three-mode processes (forbidden by the energy conservation) on the four-mode ones $[5,7]$ and also other four-mode processes, which are not directly responsible for the occurrence of the instability, so Eq. (33) is only approximate.

Three remarks are necessary. First, the assumption that only one pair of additional modes is excited at the threshold is of no physical importance, because the instability occurs when the pair (or infinitely many pairs, (cf. [8])) with the lowest threshold becomes excited, and this value of the rf field amplitude is measured as the critical one. Second, it is possible to consider the non-degenerate excitation of mode pairs with even great detunings [14,34], and in thin plates there exist an experimental evidence that the threshold power for this process may be less than for the decay of the pumped mode into a pair of degenerate modes with half the pumping frequency [14]. Third, small non-zero detunings of the excited modes, even if their frequencies might be degenerate, may be connected with the discreteness of the mode spectrum in a finite sample and sometimes have great influence on the behavior of the system far above the Suhl instability.

\section{Conclusions}

Although many papers have already been devoted to the question of the Suhl instabilities in thin slabs (Sec. 1.), this is, as far as we know, the first paper in which the 2nd order Suhl instability apart from two remaining ones has been discussed in terms of magnetostatic and dipole-exchange modes, with the exchange energy included in the Hamiltonian. Besides, the results of Sec. 2.1. are much more general and may serve as a tool for the investigation of the nonlinear ferromagnetic resonance in samples of various shapes and with different kinds of the pinning exchange boundary conditions (3). Other authors have investigated theoretically mainly the 1st order instability (the exceptions are [23, 24]) in thin slabs [22-29], probably because of its importance in many electronic devices [29]. The 1st order and parallel pumping instability of the Walker magnetostatic modes in a sphere 
[31] was discussed in $[4,30]$ and in a thin, axially magnetized cylinder in [34]. But very interesting results, especially in the study of auto-oscillations and chaos, were obtained in the main resonance (2nd order) instability configuration $[15,17]$.

The literature, known to us, does not provide any examples of exact measurements of the Suhl thresholds, when distinct magnetostatic modes are excited by uniform pumping in a thin plate. Preliminary results of our numerical investigation of the thresholds for the 1st order instability in the slab, magnetized parallel to the surface, show, however, that the parametric excitation of various types of volume dipole-exchange modes is possible via the decay of the volume or surface magnetostatic modes. If the pumped mode is conveniently chosen, it can decay not only into non-degenerate mode pairs, belonging to different surfaces in the energy spectrum, as in Ref. [14], but also into degenerate mode pairs with half the pumping frequency, belonging to the same energy surface. If only this latter case is considered, the threshold for the instability of modes with higher frequency (in general, surface modes) turns out to be higher than for these with lower frequency (in general, volume modes), what is in qualitative agreement with the experiment [21]. We are going to contain the results of our current investigation of both time-independent and time-dependent nonlinear effects in thin slabs above the Suhl threshold in a separate publication.

\section{References}

[1] H. Suhl, J. Phys. Chem. Solids 1, 209 (1957).

[2] R. Damon, in: Magnetism. A Treatise on the Modern Theory, Eds. G. Rado, H. Suhl, Vol. 1, Academic Press, New York, London 1963, p. 552.

[3] G. Gibson, C. Jeffries, Phys. Rev. A 29, 811 (1984).

[4] G. Wiese, H. Benner, Z. Phys. B, Condens. Malter 79, 119 (1990); G. Wiese, Z. Phys. B, Condens. Matter 82, 453 (1991).

[5] P.H. Bryant, C.D. Jeffries, K. Nakamura, Phys. Rev. A 38, 4223 (1988).

[6] E. Schlömann, Phys. Rev. 116, 828 (1959).

[7] V. Zakharov, V. Lvov, S.S. Starobinets, Usp. Fiz. Nauk 114, 609 (1974).

[8] H. Suhl, X.Y. Zhang, Phys. Rev. B 38, 4893 (1987).

[9] J.B. Comley, T. Penney, R.V. Jones, J. Appl. Phys. 34, 1145 (1963).

[10] J.B. Comley, R.V. Jones, J. Appl. Phys. 36, 1201 (1965).

[11] A.J. Bertraud, H. Pascard, J. Appl. Phys. 37, 2035 (1966).

[12] O.A. Chivilieva, A.G. Gurevich, A.N. Anisimov, B.N. Gusev, G.A Vugal'ter, E.S. Sher, Fiz. Tverd. Tela 29, 1774 (1987).

[13] G.A. Melkov, S.V. Sholom, Fiz. Tverd. Tela 29, 3257 (1987).

[14] G.A. Melkov, S.V. Sholom, Zh. Eksp. Teor. Fiz. 96, 712 (1989).

[15] G.M. Dudko, G.T. Kazakov, A.V. Kozhevnikov, Yu.A. Filimonov, Pis'ma Zh. Tekh. Fiz. 13, 736 (1987).

[16] P.E. Wigen, H. Doetsch, Y. Ming, L. Baselgia, F. Waldner, J. Appl. Phys 63, 4157 (1988).

[17] R.D. McMichael, P.E. Wigen, J. Appl. Phys. 64, 5474 (1988); Phys. Rev. Lett. 64, 64 (1990); Phys. Rev. B 42, 6723 (1990). 
[18] G. Srinivasan, M. Chen, C.E. Patton, J. Appl. Phys. 64, 5480 (1988).

[19] G. Srinivasan, J. Appl. Phys. 69, 5730 (1991).

[20] G. Srinivasan, Solid State Commun. 80, 5 (1991).

[21] T.L. Carroll, L.M. Peccora, F.J. Rachford, J. Appl. Phys. 67, 5631 (1990); J. Appl. Phys. 69, 5727 (1991); J. Appl. Phys. 70, 3197 (1991); IEEE.Trans. Magn. 27, 5441 (1991).

[22] V.P. Lyukomskij, A.V. Kuz'ko, Fiz. Tverd. Tela 11, 2951 (1969).

[23] Yu.V. Gulyaev, P.E. Zil'berman, S.A. Nikitov, A.G. Termiryazev, Fiz. Tverd. Tela 29, 1794 (1987).

[24] A.D. Boardman, S.A. Nikitov, Phys. Rev. B 38, 11444 (1988).

[25] O.G. Vendik, V.A. Kalinikos, D.N. Chartoryzhskij, Fiz. Tverd. Tela 19, 387 (1977).

[26] V.A. Kallinikos, Pis'ma Zh. Tekh. Fiz. 9, 811 (1983).

[27] B.A. Kallinikos, A.N. Slavin, IEEE Trans. Magn. 27, 5444 (1991).

[28] G.A. Melkov, Fiz. Tverd. Tela 30, 2533 (1988).

[29] S.N. Sitizer, P.R. Emtage, Circuits Syst. Signal Process. 4, 227 (1985).

[30] P.H. Bryant, Phys. Rev. B 39, 4363 (1989).

[31] L.R. Walker, Phys. Rev. 105, 390 (1957).

[32] R.E. De Wames, J. Wolfram, J. Appl. Phys. 41, 987 (1970).

[33] M. Sparks, Phys. Rev. B 1, 3831 (1970).

[34] E. Schlömann, R.I. Joseph, J. Appl. Phys. 32, 1006 (1961).

[35] R.W. Damon, J.R. Eschbach, J. Phys. Chem. Solids 19, 308 (1961).

[36] A.I. Akhiezer, V.G. Bar'yakhtar, S.V. Peletminskij, Spinovye volny, Nauka, Moscow 1968 (in Russian). 